\title{
On Partition Dimension of Some Cycle-Related Graphs
}

\author{
Changcheng Wei, ${ }^{1}$ Muhammad Faisal Nadeem $\mathbb{D}^{2},{ }^{2}$ Hafiz Muhammad Afzal Siddiqui ${ }^{(D},{ }^{2}$ \\ Muhammad Azeem (iD, ${ }^{2,3}$ Jia-Bao Liu $\mathbb{D}^{4}{ }^{4}$ and Adnan Khalil ${ }^{2}$
}

${ }^{1}$ Department of Mathematics and Computer Science, Tongling University, Tongling, Anhui, China

${ }^{2}$ Department of Mathematics, COMSATS University Islamabad, Lahore Campus, Lahore, Pakistan

${ }^{3}$ Department of Aerospace Engineering, Faculty of Engineering, Universiti Putra Malaysia, Seri Kembangan, Malaysia

${ }^{4}$ School of Mathematics and Physics, Anhui Jianzhu University, Hefei 230601, China

Correspondence should be addressed to Muhammad Faisal Nadeem; mfaisalnadeem@ymail.com

Received 21 August 2020; Revised 28 December 2020; Accepted 22 February 2021; Published 8 March 2021

Academic Editor: Isabella Torcicollo

Copyright (c) 2021 Changcheng Wei et al. This is an open access article distributed under the Creative Commons Attribution License, which permits unrestricted use, distribution, and reproduction in any medium, provided the original work is properly cited.

\begin{abstract}
Let $G$ be a simple connected graph. Suppose $\Delta=\left\{\Delta_{1}, \Delta_{2}, \ldots, \Delta_{l}\right\}$ an l-partition of $V(G)$. A partition representation of a vertex $\alpha$ w.r.t $\Delta$ is the $l$-vector $\left(d\left(\alpha, \Delta_{1}\right), d\left(\alpha, \Delta_{2}\right), \ldots, d\left(\alpha, \Delta_{l}\right)\right)$, denoted by $r(\alpha \mid \Delta)$. Any partition $\Delta$ is referred as resolving partition if $\forall \alpha_{i} \neq \alpha_{j} \in V(G)$ such that $r\left(\alpha_{i} \mid \Delta\right) \neq r\left(\alpha_{j} \mid \Delta\right)$. The smallest integer $l$ is referred as the partition dimension $\operatorname{pd}(G)$ of $G$ if the $l$-partition $\Delta$ is a resolving partition. In this article, we discuss the partition dimension of kayak paddle graph, cycle graph with chord, and a graph generated by chain of cycles. It has been shown that the partition dimension of the said families of graphs is constant.
\end{abstract}

\section{Introduction and Preliminaries}

The concept of partition dimension is a natural generalization of metric dimension. It was proposed in [1]. This concept came from the study of metric dimension which was defined independently in $[2,3]$. This parameter of a graph was proved NP-complete problem given [4]. The metric dimension of a connected graph is based on the distance between vertices, while partition dimension is based on the distance between a vertex and a set containing some vertices. Many researchers worked on this topic. A significant number of papers were published and some of them are given in [5-9]. The problem of finding partition dimension of a connected graph is still unsolved. There are only some lower and upper bounds for the partition dimension of general connected graph available in the literature and the exact values are still open. An upper bound for the partition dimension of a tree is given in [10]. The partition dimension of certain classes of graphs is given in [11-13].
Graph theory is very vast field of applied and computational mathematics. That is why most of the applied sciences field extensively uses the graph theory. The partition dimension is also considered an applied topic of graph theory, and some of them are Djokovic-Winkler relation [14], strategies for the mastermind game [15], network discovery and verification [16], and in chemistry to represent the chemical compounds $[17,18]$. In digital world, it is used to recognize the pattern, in robotics, it used for image processing, and it also plays a key role for the management of hierarchical data structures [19]. However, the applications of partition are still limited because the computational cost to compute the partition dimension is very complex. Some other applications of this concept is the navigation of robots in networks, and for interest, few areas appear in these literature $[2,20]$.

The partition dimension of a graph can be constant, but some graphs have bounded. Here, are some literature works. Ahmed et al. [21] computed the metric dimension of kayak paddle graph and cycle graph with chords and 
proved that adding an edge in a cycle graph will not affect the metric dimension of a cycle graph. Mehreen et al. [5] computed the partition dimension of fullerene graph is 3 . Rajan et al. [7] described the constant partition dimension of hexagonal and honeycomb networks. Partition dimension of certain honeycomb-derived networks is computed in [6]. The partition dimension of some wheel-related graphs is computed in [22]. Rodrìguez-Velàzquez et al. $[8,9]$ worked on the trees and unicyclic graphs and computed the bounds on the partition dimension. For more details, we refer to [23-26].

The distance between two vertices $v_{1}, v_{2} \in V(G)$ is a shortest path between them and is denoted by $d\left(v_{1}, v_{2}\right)$. Let $\Delta=\left\{\Delta_{1}, \ldots, \Delta_{l}\right\}$ be an $l$-partition of the vertex set $V(G)$ and $r(v \mid \Delta)=\left\{d\left(v, \Delta_{1}\right), d\left(v, \Delta_{2}\right), \ldots, d\left(v, \Delta_{a}\right)\right\}$ be an l-tuple representing a unique code of $v$ w.r.t to $\Delta$. If all representation codes of the vertex set of the graph $G$ are unique w.r.t to $\Delta$, then $\Delta$ is a resolving partition. This partition with the minimum value of $l$ is referred as partition dimension of $G$ and is denoted by pd $(G)$.

This paper deals with the partition dimension of the kayak paddle graph, a cycle graph with a chord, and a graph generated by a chain of cycles. More precisely, we computed the partition dimension of said families of graphs and it turns out to be constant, i.e., 3 .

Following theorems are very helpful in finding the partition dimension of a connected graph.
Theorem 1 (see [1]). Let $\Pi$ be a resolving partition of $V(G)$ of a graph $G$ and $u, v \in V(G)$. If $d(u, w)=d(v, w)$ for all vertices $w \in V(G) /(u, v)$, then $u$ and $v$ belong to different classes of $\Pi$.

Theorem 2 (see [1]). Let $G$ be a simple and connected graph of order $n$; then,

(i) $\operatorname{pd}(G)$ is 2 iff $G$ is a path graph

(ii) $p d(G)$ is $n$ iff $G$ is a complete graph

The remaining article is managed as follows. In Section 2, the partition dimension of kayak paddle graph is computed, which is constant for all three parameters given in the definition. In Section 3, the partition dimension of cycle graph with chord is discussed, and in Section 4, we study partition dimension of a family of graph generated by chain of cycles. In the end, conclusion and references have been given.

\section{Kayak Paddle Graph}

This section deals with the graph generated by cycle, known as kayak paddle graph, denoted by $\operatorname{KP}(x, y, z)$. This family of graphs can be constructed by considering two cycles of length $x$ and $y$ and a path of length $z$ with $x, y \geq 3$ and $z \geq 2$. The vertex set and edge set of kayak paddle graph are as follows, respectively:

$$
\begin{aligned}
& V(\operatorname{KP}(x, y, z))=\left\{\alpha_{1}, \alpha_{2}, \ldots, \alpha_{x}\right\} \cup\left\{\beta_{1}, \beta_{2}, \ldots, \beta_{y}\right\} \cup\left\{\gamma_{1}, \ldots, \gamma_{z-1}\right\}, \\
& E(\operatorname{KP}(x, y, z))=\left\{\alpha_{i} \alpha_{i+1}: 1 \leq i \leq x\right\} \cup\left\{\beta_{i} \beta_{i+1}: 1 \leq i \leq y\right\} \cup\left\{\gamma_{i} \gamma_{i+1}: 1 \leq i \leq z-2\right\} \cup\left\{\alpha_{1} \gamma_{1}, \gamma_{z-1} \beta_{1}\right\},
\end{aligned}
$$

where $\alpha_{x+1}=\alpha_{1}$ and $\beta_{1}=\beta_{y+1}$.

Figure 1 shows a kayak paddle graph $\operatorname{KP}(12,8,5)$ with two cycles of length $x=12$ and $y=8$ and a path of length $z=5$ joining them.

2.1. Partition Dimension of Kayak Paddle Graph. In the following result, partition dimension of the kayak paddle graph generated by two cycles and a path graph has been discussed.

Theorem 3. Let $G=K P(x, y, z)$ be a kayak paddle graph with $x \geq 3, y \geq 4$, and $z \geq 2$. Then, $p d(K P(x, y, z))=3$.

Proof. To show that $\operatorname{pd}(\operatorname{KP}(x, y, z))=3$, we use double inequality. First of all, we prove that $\operatorname{pd}(\operatorname{KP}(x, y, z)) \leq 3$ by constructing a resolving partition with three elements. For this, consider the following cases on $x$ and $y$ : (i) Case 1: when $x=2 s$ and $y=2 t$ and $s, t \geq 2$. We claim $\Delta=\left\{\Delta_{1}, \Delta_{2}, \Delta_{3}\right\}$ is a resolving set, where $\Delta_{1}=\left\{\alpha_{s}\right\}, \Delta_{2}=\left\{\beta_{t}\right\}$, and $\Delta_{3}=\left\{q \in V(G) \mid q \notin \Delta_{1}\right.$, $\left.\Delta_{2}\right\}$, where $q$ is any of the vertex of the type $\alpha_{i}, \beta_{i}$, or $\gamma_{i}$ other than the vertices of $\Delta_{1}$ and $\Delta_{2}$. Then, Table 1 shows different representations of $\alpha_{\varepsilon}, \beta_{\varepsilon}$, and $\gamma_{\varepsilon}$ vertices with respect to resolving partition $\Delta$, where $a=1$ when $\varepsilon=s, b=1$ when $\varepsilon=t$, and $a, b=0$, otherwise.

(iii) Case 2: when $x=2 s+1, y=2 t$ and $t \geq 2$. Then, we split $x$ into the following three subcases:

Subcase 2.1: for $x=3,5$, we claim $\Delta=\left\{\Delta_{1}, \Delta_{2}, \Delta_{3}\right\}$ is a resolving partition with $\Delta_{1}=\left\{\alpha_{2}\right\}, \Delta_{2}=\left\{\beta_{t}\right\}$, and $\Delta_{3}=\left\{q \in V(G) \mid q \notin \Delta_{1}, \Delta_{2}\right\}$. Then, Table 2 shows different representations of $\alpha_{\varepsilon}, \beta_{\varepsilon}$, and $\gamma_{\varepsilon}$ with respect to partition $\Delta$, where $a=1$ when $\varepsilon=2, b=1$ when $\varepsilon=t$, and $a, b=0$, otherwise. Subcase 2.2: for $x=7$, we claim $\Delta=\left\{\Delta_{1}, \Delta_{2}, \Delta_{3}\right\}$ is a resolving partition with $\Delta_{1}=\left\{\alpha_{3}\right\}, \Delta_{2}=\left\{\beta_{t}\right\}$, and 


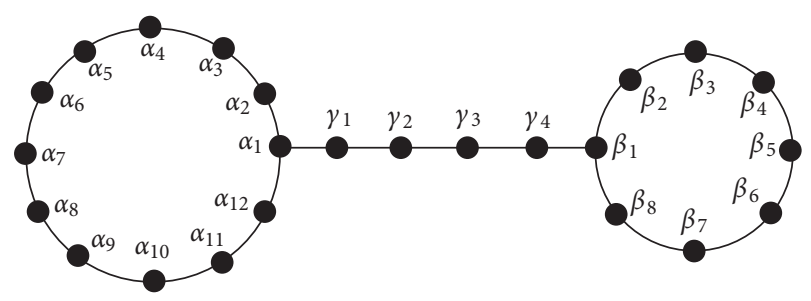

Figure 1: A kayak paddle graph: $\operatorname{KP}(12,8,5)$.

TABLE 1: Distance codes for the vertices of $\operatorname{KP}(x, y, z)$ w.r.t $\Delta$.

\begin{tabular}{lccc}
\hline$d(\cdot, \cdot)$ & $\Delta_{1}$ & $\Delta_{2}$ & $\Delta_{3}$ \\
\hline$\alpha_{\varepsilon}: 1 \leq \varepsilon \leq s$ & $s-\varepsilon$ & $t+z-2+\varepsilon$ & $a$ \\
$\alpha_{\varepsilon}: s+1 \leq \varepsilon \leq x$ & $\varepsilon-s$ & $2 s+t+z-\varepsilon$ & 0 \\
$\beta_{\varepsilon}: 1 \leq \varepsilon \leq t$ & $s+z-2+\varepsilon$ & $t-\varepsilon$ & $b$ \\
$\beta_{\varepsilon}: t+1 \leq \varepsilon \leq x$ & $2 t+s+z-\varepsilon$ & $\varepsilon-t$ & 0 \\
$\gamma_{\varepsilon}: 1 \leq \varepsilon \leq z-1$ & $s+\varepsilon-1$ & $t-\varepsilon+z-1$ & 0 \\
\hline
\end{tabular}

TABLE 2: Distance codes for the vertices of $\operatorname{KP}(x, y, z)$ w.r.t $\Delta$.

\begin{tabular}{lccc}
\hline$d(\cdot, \cdot)$ & $\Delta_{1}$ & $\Delta_{2}$ & $\Delta_{3}$ \\
\hline$\alpha_{\varepsilon}: \varepsilon=1,2$ & $2-\varepsilon$ & $t+z-2+\varepsilon$ & $a$ \\
$\alpha_{\varepsilon}: \varepsilon=x=3$ & 1 & $t+z$ & 0 \\
$\alpha_{\varepsilon}: \varepsilon=3,4, x=5$ & $\varepsilon-2$ & $t+z+1$ & 0 \\
$\alpha_{\varepsilon}: \varepsilon=x=5$ & 2 & $t+z$ & 0 \\
$\beta_{\varepsilon}: 1 \leq \varepsilon \leq t$ & $z+\varepsilon$ & $t-\varepsilon$ & $b$ \\
$\beta_{\varepsilon}: t+1 \leq \varepsilon \leq y$ & $2 t+2+z-\varepsilon$ & $\varepsilon-t$ & 0 \\
$\gamma_{\varepsilon}: 1 \leq \varepsilon \leq z-1$ & $1+\varepsilon$ & $t-\varepsilon+z-1$ & 0 \\
\hline
\end{tabular}

$\Delta_{3}=\left\{q \in V(G) \mid q \notin \Delta_{1}, \Delta_{2}\right\}$. Then, Table 3 shows different representations of $\alpha_{\varepsilon}, \beta_{\varepsilon}$, and $\gamma_{\varepsilon}$ with respect to the partition $\Delta$, where $a=1$ when $\varepsilon=3$, $b=1$ when $\varepsilon=t$, and $a, b=0$, otherwise.

Subcase 2.3: for $x=2 s+1$ and $s \geq 4$, we claim $\Delta=$ $\left\{\Delta_{1}, \Delta_{2}, \Delta_{3}\right\}$ is a resolving partition $\Delta_{1}=\left\{\alpha_{s}\right\}$, $\Delta_{2}=\left\{\beta_{t}\right\}$, and $\Delta_{3}=\left\{q \in V(G) \mid q \notin \Delta_{1}, \Delta_{2}\right\}$. Then, Table 4 shows different representations of $\alpha_{\varepsilon}, \beta_{\varepsilon}$, and $\gamma_{\varepsilon}$ with respect to the partition $\Delta$, where $a=1$ when $\varepsilon=s, b=1$ when $\varepsilon=t$, and $a, b=0$, otherwise.

(iv) Case 3: when $x=2 s+1$ and $y=2 t+1$, then we split $x$ and $y$ in the following 9 subcases:

Subcase 3.1: for $x=3$ and $y=3,5$, we claim $\Delta=$ $\left\{\Delta_{1}, \Delta_{2}, \Delta_{3}\right\}$ is a resolving partition with $\Delta_{1}=\left\{\alpha_{2}\right\}$, $\Delta_{2}=\left\{\beta_{2}\right\}$, and $\Delta_{3}=\left\{q \in V(G) \mid q \notin \Delta_{1}, \Delta_{2}\right\}$. Then, Table 5 shows different representations of $\alpha_{\varepsilon}, \beta_{\varepsilon}$, and $\gamma_{\varepsilon}$ with respect to the partition $\Delta$, where $a=$ $b=1$ when $\varepsilon=2$ and $a, b=0$, otherwise.

Subcase 3.2: for $x=3$ and $y=7$, we claim $\Delta=$ $\left\{\Delta_{1}, \Delta_{2}, \Delta_{3}\right\}$ is a resolving partition with $\Delta_{1}=\left\{\alpha_{2}\right\}$, $\Delta_{2}=\left\{\beta_{3}\right\}, \Delta_{3}=\left\{q \in V(G) \mid q \notin \Delta_{1}, \Delta_{2}\right\}$. Then, Table 6 shows different representations of $\alpha_{\varepsilon}, \beta_{\mathcal{\varepsilon}}$, and $\gamma_{\varepsilon}$ with respect to the partition $\Delta$, where $a=1$
TABle 3: Distance codes for the vertices of $\operatorname{KP}(x, y, z)$ w.r.t $\Delta$.

\begin{tabular}{lccc}
\hline$d(\cdot, \cdot)$ & $\Delta_{1}$ & $\Delta_{2}$ & $\Delta_{3}$ \\
\hline$\alpha_{\varepsilon}: \varepsilon=1,2,3$ & $3-\varepsilon$ & $t+z-2+\varepsilon$ & $a$ \\
$\alpha_{\varepsilon}: \varepsilon=4,5$ & $\varepsilon-3$ & $t+z+2$ & 0 \\
$\alpha_{\varepsilon}: \varepsilon=6,7$ & 3 & $t+z-\varepsilon+7$ & 0 \\
$\beta_{\varepsilon}: 1 \leq \varepsilon \leq t$ & $z+\varepsilon+1$ & $t-\varepsilon$ & $b$ \\
$\beta_{\varepsilon}: t+1 \leq \varepsilon \leq y$ & $2 t+z-\varepsilon+3$ & $\varepsilon-t$ & 0 \\
$\gamma_{\varepsilon}: 1 \leq \varepsilon \leq z-1$ & $\varepsilon+2$ & $t-\varepsilon+z-1$ & 0 \\
\hline
\end{tabular}

TABLE 4: Distance codes for the vertices of $\operatorname{KP}(x, y, z)$ w.r.t $\Delta$.

\begin{tabular}{lccc}
\hline$d(\cdot, \cdot)$ & $\Delta_{1}$ & $\Delta_{2}$ & $\Delta_{3}$ \\
\hline$\alpha_{\varepsilon}: 1 \leq \varepsilon \leq s$ & $s-\varepsilon$ & $t+z-2+\varepsilon$ & $a$ \\
$\alpha_{\varepsilon}: \varepsilon=s+1, s+2$ & $\varepsilon-s$ & $s+t+z-1$ & 0 \\
$\alpha_{\varepsilon}: s+3 \leq \varepsilon \leq 2 s-1$ & $\varepsilon-s$ & $2 s+t+z-\varepsilon+1$ & 0 \\
$\alpha_{\varepsilon}: 2 s \leq \varepsilon \leq x$ & $s$ & $2 s+t+z-\varepsilon+1$ & 0 \\
$\beta_{\varepsilon}: 1 \leq \varepsilon \leq t$ & $s+z+\varepsilon-2$ & $t-\varepsilon$ & $b$ \\
$\beta_{\varepsilon}: t+1 \leq \varepsilon \leq y$ & $2 t+s+z-\varepsilon$ & $\varepsilon-t$ & 0 \\
$\gamma_{\varepsilon}: 1 \leq \varepsilon \leq z-1$ & $\varepsilon+s-1$ & $t-\varepsilon+z-1$ & 0 \\
\hline
\end{tabular}

TABLE 5: Distance codes for the vertices of $\operatorname{KP}(x, y, z)$ w.r.t $\Delta$.

\begin{tabular}{lccc}
\hline$d(\cdot, \cdot)$ & $\Delta_{1}$ & $\Delta_{2}$ & $\Delta_{3}$ \\
\hline$\alpha_{\varepsilon}: \varepsilon=1,2$ & $2-\varepsilon$ & $z+\varepsilon$ & $a$ \\
$\alpha_{\varepsilon}: \varepsilon=3$ & 1 & $z+2$ & 0 \\
$\beta_{\varepsilon}: \varepsilon=1,2$ & $z+\varepsilon$ & $2-\varepsilon$ & $b$ \\
$\beta_{\varepsilon}: \varepsilon=y=3$ & $z+2$ & 1 & 0 \\
$\beta_{\varepsilon}: \varepsilon=3,4, y=5$ & $z+3$ & $\varepsilon-2$ & 0 \\
$\beta_{\varepsilon}: \varepsilon=y=5$ & $z+2$ & 2 & 0 \\
$\gamma_{\varepsilon}: 1 \leq \varepsilon \leq z-1$ & $\varepsilon+1$ & $1-\varepsilon+z$ & 0 \\
\hline
\end{tabular}

TABLe 6: Distance codes for the vertices of $\operatorname{KP}(x, y, z)$ w.r.t $\Delta$.

\begin{tabular}{lccc}
\hline$d(\cdot, \cdot)$ & $\Delta_{1}$ & $\Delta_{2}$ & $\Delta_{3}$ \\
\hline$\alpha_{\varepsilon}: \varepsilon=1,2$ & $2-\varepsilon$ & $z+\varepsilon+1$ & $a$ \\
$\alpha_{\varepsilon}: \varepsilon=3$ & 1 & $z+3$ & 0 \\
$\beta_{\varepsilon}: \varepsilon=1,2,3$ & $z+\varepsilon$ & $3-\varepsilon$ & $b$ \\
$\beta_{\varepsilon}: \varepsilon=4,5$ & $z+4$ & $\varepsilon-3$ & 0 \\
$\beta_{\varepsilon}: \varepsilon=6,7$ & $z+9-\varepsilon$ & 3 & 0 \\
$\gamma_{\varepsilon}: 1 \leq \varepsilon \leq z-1$ & $\varepsilon+1$ & $2-\varepsilon+z$ & 0 \\
\hline
\end{tabular}

when $\varepsilon=2, b=1$ when $\varepsilon=3$, and $a, b=0$, otherwise.

Subcase 3.3: for $x=3$ and $y=2 t+1$ and $t \geq 4$, we claim $\Delta=\left\{\Delta_{1}, \Delta_{2}, \Delta_{3}\right\}$ is a resolving partition, where $\Delta_{1}=\left\{\alpha_{2}\right\}, \Delta_{2}=\left\{\beta_{t}\right\}$, and $\Delta_{3}=\{q \in V(G) \mid$ $\left.q \notin \Delta_{1}, \Delta_{2}\right\}$. Then, Table 7 shows different representations of $\alpha_{\varepsilon}, \beta_{\varepsilon}$, and $\gamma_{\varepsilon}$ with respect to the partition $\Delta$, where $a=1$ when $\varepsilon=2, b=1$ when $\varepsilon=t$, and $a, b=0$, otherwise.

Subcase 3.4: for $x=5$ and $y=5$ and $t \geq 4$, we claim $\Delta=\left\{\Delta_{1}, \Delta_{2}, \Delta_{3}\right\}$ is a resolving partition, where $\Delta_{1}=\left\{\alpha_{2}\right\}, \Delta_{2}=\left\{\beta_{2}\right\}$, and $\Delta_{3}=\left\{q \in V(G) \mid q \notin \Delta_{1}\right.$, $\left.\Delta_{2}\right\}$. Then, Table 8 shows different representation of $\alpha_{\varepsilon}, \beta_{\varepsilon}$, and $\gamma_{\varepsilon}$ with respect to the partition $\Delta$, 
TABLE 7: Distance codes for the vertices of $\operatorname{KP}(x, y, z)$ w.r.t $\Delta$.

\begin{tabular}{lccc}
\hline$d(\cdot, \cdot)$ & $\Delta_{1}$ & $\Delta_{2}$ & $\Delta_{3}$ \\
\hline$\alpha_{\varepsilon}: \varepsilon=1,2$ & $2-\varepsilon$ & $t+z+\varepsilon-2$ & $a$ \\
$\alpha_{\varepsilon}: \varepsilon=3$ & 1 & $t+z$ & 0 \\
$\beta_{\varepsilon}: 1 \leq \varepsilon \leq t$ & $z+\varepsilon$ & $t-\varepsilon$ & $b$ \\
$\beta_{\varepsilon}: \varepsilon=t+1, t+2$ & $t+z+1$ & $\varepsilon-t$ & 0 \\
$\beta_{\varepsilon}: t+3 \leq \varepsilon \leq 2 t-1$ & $2 t+z-\varepsilon+3$ & $\varepsilon-t$ & 0 \\
$\beta_{\varepsilon}: 2 t \leq \varepsilon \leq y$ & $2 t+z-\varepsilon+3$ & $t$ & 0 \\
$\gamma_{\varepsilon}: 1 \leq \varepsilon \leq z-1$ & $1+\varepsilon$ & $t-\varepsilon+z-1$ & 0 \\
\hline
\end{tabular}

TABLE 8: Distance codes for the vertices of $\operatorname{KP}(x, y, z)$ w.r.t $\Delta$.

\begin{tabular}{lccc}
\hline$d(\cdot, \cdot)$ & $\Delta_{1}$ & $\Delta_{2}$ & $\Delta_{3}$ \\
\hline$\alpha_{\varepsilon}: \varepsilon=1,2$ & $2-\varepsilon$ & $z+\varepsilon$ & $a$ \\
$\alpha_{\varepsilon}: \varepsilon=3,4$ & $\varepsilon-1$ & $z+3$ & 0 \\
$\alpha_{\varepsilon}: \varepsilon=5$ & 2 & $z+2$ & 0 \\
$\beta_{\varepsilon}: \varepsilon=1,2$ & $z+\varepsilon$ & $2-\varepsilon$ & $b$ \\
$\beta_{\varepsilon}: \varepsilon=3,4$ & $z+3$ & $\varepsilon-2$ & 0 \\
$\beta_{\varepsilon}: \varepsilon=5$ & $2+z$ & 2 & 0 \\
$\gamma_{\varepsilon}: 1 \leq \varepsilon \leq z-1$ & $1+\varepsilon$ & $z-\varepsilon+1$ & 0 \\
\hline
\end{tabular}

where $a=b=1$ when $\varepsilon=2$ and otherwise $a, b$ are 0 .

Subcase 3.5: for $x=5$ and $y=7$ and $t \geq 4$, we claim $\Delta=\left\{\Delta_{1}, \Delta_{2}, \Delta_{3}\right\}$ is a resolving partition, where $\Delta_{1}=\left\{\alpha_{2}\right\}, \Delta_{2}=\left\{\beta_{3}\right\}$, and $\Delta_{3}=\left\{q \in V(G) \mid q \notin \Delta_{1}\right.$, $\left.\Delta_{2}\right\}$. Then, Table 9 shows different representation of $\alpha_{\varepsilon}, \beta_{\varepsilon}$, and $\gamma_{\varepsilon}$ with respect to the partition $\Delta$, where $a=1$ when $\varepsilon=2, b=1$ when $\varepsilon=3$, and $a, b=0$, otherwise.

Subcase 3.6: for $x=5$ and $y=2 t+1$ and $t \geq 4$, we claim $\Delta=\left\{\Delta_{1}, \Delta_{2}, \Delta_{3}\right\}$ is a resolving partition, where $\Delta_{1}=\left\{\alpha_{2}\right\}, \Delta_{2}=\left\{\beta_{t}\right\}$, and $\Delta_{3}=\{q \in V(G) \mid$ $\left.q \notin \Delta_{1}, \Delta_{2}\right\}$. Then, Table 10 shows different representation of $\alpha_{\varepsilon}, \beta_{\varepsilon}$, and $\gamma_{\varepsilon}$ with respect to the partition $\Delta$, where $a=1$ when $\varepsilon=2, b=1$ when $\varepsilon=t$, and $a, b=0$, otherwise.

Subcase 3.7: for $x=7$ and $y=7$, we claim $\Delta=$ $\left\{\Delta_{1}, \Delta_{2}, \Delta_{3}\right\}$ is a resolving partition, where $\Delta_{1}=\left\{\alpha_{3}\right\}, \Delta_{2}=\left\{\beta_{3}\right\}$, and $\Delta_{3}=\left\{q \in V(G) \mid q \notin \Delta_{1}\right.$, $\left.\Delta_{2}\right\}$. Then, Table 11 shows different representations of $\alpha_{\varepsilon}, \beta_{\varepsilon}$, and $\gamma_{\varepsilon}$ with respect to the partition $\Delta$, where $a=b=1$ when $\varepsilon=3$ and $a, b=0$, otherwise.

Subcase 3.8: for $x=7$ and $y=2 t+1$ and $t \geq 4$, we claim $\Delta=\left\{\Delta_{1}, \Delta_{2}, \Delta_{3}\right\}$ is a resolving with $\Delta_{1}=\left\{\alpha_{3}\right\}, \Delta_{2}=\left\{\beta_{t}\right\}$, and $\Delta_{3}=\left\{q \in V(G) \mid q \notin \Delta_{1}\right.$, $\left.\Delta_{2}\right\}$. Then, Table 12 shows different representations of $\alpha_{\varepsilon}, \beta_{\varepsilon}$, and $\gamma_{\varepsilon}$ with respect to the partition $\Delta$, where $a=1$ when $\varepsilon=3, b=1$ when $\varepsilon=t$, and $a, b=0$, otherwise.

Subcase 3.9: for $x=2 s+1$ and $y=2 t+1$ and $s, t \geq 4$, we claim $\Delta=\left\{\Delta_{1}, \Delta_{2}, \Delta_{3}\right\}$ is a resolving 3partition with $\Delta_{1}=\left\{\alpha_{s}\right\}, \quad \Delta_{2}=\left\{\beta_{t}\right\}$, and $\Delta_{3}=\left\{q \in V(G) \mid q \notin \Delta_{1}, \Delta_{2}\right\}$. Then, Table 13 shows different representation of $\alpha_{\varepsilon}, \beta_{\varepsilon}$, and $\gamma_{\varepsilon}$ with respect to the partition $\Delta$, where $a=1$ when $\varepsilon=s$, $b=1$ when $\varepsilon=t$, and $a, b=0$, otherwise.
TABle 9: Distance codes for the vertices of $\operatorname{KP}(x, y, z)$ w.r.t $\Delta$.

\begin{tabular}{lccc}
\hline$d(\cdot, \cdot)$ & $\Delta_{1}$ & $\Delta_{2}$ & $\Delta_{3}$ \\
\hline$\alpha_{\varepsilon}: \varepsilon=1,2$ & $2-\varepsilon$ & $z+\varepsilon+1$ & $a$ \\
$\alpha_{\varepsilon}: \varepsilon=3,4$ & $\varepsilon-1$ & $z+4$ & 0 \\
$\alpha_{\varepsilon}: \varepsilon=5$ & 2 & $z+3$ & 0 \\
$\beta_{\varepsilon}: \varepsilon=1,2,3$ & $z+\varepsilon$ & $3-\varepsilon$ & $b$ \\
$\beta_{\varepsilon}: \varepsilon=4,5$ & $z+4$ & $\varepsilon-3$ & 0 \\
$\beta_{\varepsilon}: \varepsilon=5$ & $z+9-\varepsilon$ & 3 & 0 \\
$\gamma_{\varepsilon}: 1 \leq \varepsilon \leq z-1$ & $1+\varepsilon$ & $z-\varepsilon+2$ & 0 \\
\hline
\end{tabular}

TABle 10: Distance codes for the vertices of $\operatorname{KP}(x, y, z)$ w.r.t $\Delta$.

\begin{tabular}{lccc}
\hline$d(\cdot, \cdot)$ & $\Delta_{1}$ & $\Delta_{2}$ & $\Delta_{3}$ \\
\hline$\alpha_{\varepsilon}: \varepsilon=1,2$ & $2-\varepsilon$ & $t+z+\varepsilon-2$ & $a$ \\
$\alpha_{\varepsilon}: \varepsilon=3,4$ & $\varepsilon-1$ & $t+z+1$ & 0 \\
$\alpha_{\varepsilon}: \varepsilon=5$ & 2 & $z+t$ & 0 \\
$\beta_{\varepsilon}: 1 \leq \varepsilon \leq t$ & $z+\varepsilon$ & $t-\varepsilon$ & $b$ \\
$\beta_{\varepsilon}: \varepsilon=t+1, t+2$ & $t+z+1$ & $\varepsilon-t$ & 0 \\
$\beta_{\varepsilon}: t+3 \leq \varepsilon \leq 2 t-1$ & $2 t+z-\varepsilon+3$ & $t$ & 0 \\
$\gamma_{\varepsilon}: 1 \leq \varepsilon \leq z-1$ & $1+\varepsilon$ & $z-\varepsilon+1$ & 0 \\
\hline
\end{tabular}

TABLE 11: Distance codes for the vertices of $\operatorname{KP}(x, y, z)$ w.r.t $\Delta$.

\begin{tabular}{lccc}
\hline$d(\cdot, \cdot)$ & $\Delta_{1}$ & $\Delta_{2}$ & $\Delta_{3}$ \\
\hline$\alpha_{\varepsilon}: \varepsilon=1,2,3$ & $3-\varepsilon$ & $z+\varepsilon+1$ & $a$ \\
$\alpha_{\varepsilon}: \varepsilon=4,5$ & $\varepsilon-3$ & $z+5$ & 0 \\
$\alpha_{\varepsilon}: \varepsilon=6,7$ & 3 & $z+10-\varepsilon$ & 0 \\
$\beta_{\varepsilon}: \varepsilon=1,2,3$ & $z+1+\varepsilon$ & $3-\varepsilon$ & $b$ \\
$\beta_{\varepsilon}: \varepsilon=4,5$ & $z+5$ & $\varepsilon-3$ & 0 \\
$\beta_{\varepsilon}: \varepsilon=6,7$ & $z+10-\varepsilon$ & 3 & 0 \\
$\gamma_{\varepsilon}: 1 \leq \varepsilon \leq z-1$ & $2+\varepsilon$ & $z-\varepsilon+2$ & 0 \\
\hline
\end{tabular}

TABLE 12: Distance codes for the vertices of $\operatorname{KP}(x, y, z)$ w.r.t $\Delta$.

\begin{tabular}{lccc}
\hline$d(\cdot, \cdot)$ & $\Delta_{1}$ & $\Delta_{2}$ & $\Delta_{3}$ \\
\hline$\alpha_{\varepsilon}: \varepsilon=1,2,3$ & $3-\varepsilon$ & $t+z+\varepsilon-2$ & $a$ \\
$\alpha_{\varepsilon}: \varepsilon=4,5$ & $\varepsilon-3$ & $t+z+2$ & 0 \\
$\alpha_{\varepsilon}: \varepsilon=6,7$ & 3 & $t+z-\varepsilon+7$ & 0 \\
$\beta_{\varepsilon}: 1 \leq \varepsilon \leq t$ & $z+1+\varepsilon$ & $t-\varepsilon$ & $b$ \\
$\beta_{\varepsilon}: \varepsilon=t+1, t+2$ & $t+z+2$ & $\varepsilon-t$ & 0 \\
$\beta_{\varepsilon}: t+3 \leq \varepsilon \leq 2 t-1$ & $2 t+z-\varepsilon+4$ & $\varepsilon-t$ & 0 \\
$\beta_{\varepsilon}: 2 t \leq \varepsilon \leq y$ & $2 t+z-\varepsilon+4$ & $t$ & 0 \\
$\gamma_{\varepsilon}: 1 \leq \varepsilon \leq z-1$ & $2+\varepsilon$ & $t+z-\varepsilon-1$ & 0 \\
\hline
\end{tabular}

TABLE 13: Distance codes for the vertices of $\operatorname{KP}(x, y, z)$ w.r.t $\Delta$.

\begin{tabular}{lccc}
\hline$d(\cdot, \cdot)$ & $\Delta_{1}$ & $\Delta_{2}$ & $\Delta_{3}$ \\
\hline$\alpha_{\varepsilon}: 1 \leq \varepsilon \leq s$ & $s-\varepsilon$ & $t+z+\varepsilon-2$ & $a$ \\
$\alpha_{\varepsilon}: \varepsilon=s+1, s+2$ & $\varepsilon-s$ & $t+s+z-1$ & 0 \\
$\alpha_{\varepsilon}: s+3 \leq \varepsilon \leq 2 s-1$ & $\varepsilon-s$ & $2 s+t+z-\varepsilon+1$ & 0 \\
$\alpha_{\varepsilon}: 2 s \leq \varepsilon \leq x$ & $s$ & $2 s+t+z-\varepsilon+1$ & 0 \\
$\beta_{\varepsilon}: 1 \leq \varepsilon \leq t$ & $s+z-1+\varepsilon$ & $t-\varepsilon$ & $b$ \\
$\beta_{\varepsilon}: \varepsilon=t+1, t+2$ & $s+2 t+z-\varepsilon+1$ & $\varepsilon-t$ & 0 \\
$\beta_{\varepsilon}: t+3 \leq \varepsilon \leq 2 t-1$ & $s+2 t+z-\varepsilon+1$ & $\varepsilon-t$ & 0 \\
$\beta_{\varepsilon}: 2 t \leq \varepsilon \leq y$ & $s+2 t+z-\varepsilon+1$ & $t$ & 0 \\
$\gamma_{\varepsilon}: 1 \leq \varepsilon \leq z-1$ & $s-1+\varepsilon$ & $t+z-\varepsilon-1$ & 0 \\
\hline
\end{tabular}


Since all the vertices $\alpha_{\varepsilon}, \beta_{\varepsilon}$, and $\gamma_{\varepsilon}$ have different representations with respect to the partition $\Delta$, we obtain

$$
\operatorname{pd}(\mathrm{KP}(x, y, z)) \leq 3
$$

Conversely. To prove that $\operatorname{pd}(\operatorname{KP}(x, y, z)) \geq 3$, suppose, on contrary, $\operatorname{pd}(\operatorname{KP}(x, y, z))=2$. It is not possible because $\operatorname{pd}(G)=2$ if and only $G$ is a path graph. Our assumption is wrong. Therefore,

$$
\operatorname{pd}(\mathrm{KP}(x, y, z)) \geq 3 .
$$

Hence, from inequalities (2) and (3), we conclude that

$$
\operatorname{pd}(\mathrm{KP}(x, y, z))=3 \text {. }
$$

\section{Partition Dimension of Cycle with Chord Graph}

In this section, we consider a cycle with chord graph, denoted by $C_{n}^{m}$. This graph is constructed by joining any two nonadjacent vertices of the cycle. A graph of cycle with chord with vertex set $V\left(C_{n}^{m}\right)=\left\{v_{1}, v_{2}, \ldots, v_{n}\right\}$ and $E\left(C_{n}^{m}\right)=$ $\left\{v_{i} v_{i+1}: 1 \leq i \leq n\right\} \cup\left\{v_{1} v_{m+1}\right\}$ is shown in Figure 2 . To find the partition dimension of cycle with chord graph $C_{n}^{m}$, it suffices to consider $t \leq\lfloor(n / 2)\rfloor$ for given value of $n$.

The partition dimension of the cycle with chord graph is given in the following result.

Theorem 4. Let $C_{n}^{m}$ be a cycle with chord graph, $n \geq 4$ and $2 \leq m \leq n-2$. Then, $p d\left(C_{n}^{m}\right)=3$.

Proof. To show that $\operatorname{pd}\left(C_{n}^{m}\right)=3$, first, we will prove $\operatorname{pd}\left(C_{n}^{m}\right) \leq 3$ by constructing a resolving partition having 3 elements. For this, we split $n$ and $m$ into the following two cases:

(i) Case 1: for $n=2 t$ and $m=2 s$, we claim $\Delta=\left\{\Delta_{1}, \Delta_{2}, \Delta_{3}\right\}$ is a resolving partition with $\Delta_{1}=\left\{v_{t+s}\right\}, \quad \Delta_{2}=\left\{v_{t+s+1}\right\}, \quad$ and $\Delta_{3}=\left\{v_{\varepsilon} \in V(G) \mid v_{\varepsilon} \notin \Delta_{1}, \Delta_{2}\right\}$. Then, Table 14 shows different representations of $v_{\varepsilon}$ with respect to the partition $\Delta$, where $a=1$ when $\varepsilon=t+s$ and $t+s+1$ and 0 , otherwise.

(iii) Case 2: when $n=2 t+1$ and $m=2 s+1$, we claim that $\Delta=\left\{\Delta_{1}, \Delta_{2}, \Delta_{3}\right\}$ is a resolving partition with $\Delta_{1}=\left\{v_{t+s+1}\right\}, \quad \Delta_{2}=\left\{v_{t+s+2}\right\}, \quad$ and $\Delta_{3}=\left\{v_{\varepsilon} \in V(G) \mid v_{\varepsilon} \notin \Delta_{1}, \Delta_{2}\right\}$. Then, Table 15 shows different representations of $v_{\varepsilon}$ with respect to the partition $\Delta$, where $a=1$ when $\varepsilon=t+s+1$ and $t+$ $s+2$ and 0 , otherwise.

Case 2.1: when $n=2 t$ and $m=2 s+1$, where $a=1$ when $\varepsilon=t+s+1$ and $t+s+2$ and 0 , otherwise. Table 16 shows different representations of $v_{\varepsilon}$ with respect to the partition $\Delta$.

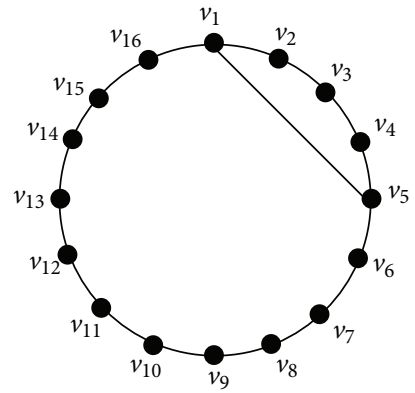

Figure 2: A cycle with chord graph $C_{16}^{4}$.

TABle 14: Distance codes for the vertices of $C_{n}^{m}$ w.r.t $\Delta$.

\begin{tabular}{lccc}
\hline$d(\cdot, \cdot)$ & $\Delta_{1}$ & $\Delta_{2}$ & $\Delta_{3}$ \\
\hline$v_{\varepsilon}: 1 \leq \varepsilon \leq s$ & $t-s-1+\varepsilon$ & $t-s+\varepsilon-1$ & 0 \\
$v_{\varepsilon}: s+1 \leq \varepsilon \leq t+s$ & $t+s-\varepsilon$ & $t+s-\varepsilon+1$ & $a$ \\
$v_{\varepsilon}: t+s+1 \leq \varepsilon \leq n$ & $\varepsilon-t-s$ & $\varepsilon-t-s-1$ & $a$ \\
\hline
\end{tabular}

TABle 15: Distance codes for the vertices of $C_{n}^{m}$ w.r.t $\Delta$.

\begin{tabular}{lccc}
\hline$d(\cdot, \cdot)$ & $\Delta_{1}$ & $\Delta_{2}$ & $\Delta_{3}$ \\
\hline$v_{\varepsilon}: 1 \leq \varepsilon \leq s+1$ & $t-s-1+\varepsilon$ & $t-s+\varepsilon-1$ & 0 \\
$v_{\varepsilon}: s+2 \leq \varepsilon \leq t+s+1$ & $t+s-\varepsilon+1$ & $t+s-\varepsilon+2$ & $a$ \\
$v_{\varepsilon}: t+s+2 \leq \varepsilon \leq n$ & $\varepsilon-t-s-1$ & $\varepsilon-t-s-2$ & $a$ \\
\hline
\end{tabular}

TABLE 16: Distance codes for the vertices of $C_{n}^{m}$ w.r.t $\Delta$.

\begin{tabular}{lccc}
\hline$d(\cdot, \cdot)$ & $\Delta_{1}$ & $\Delta_{2}$ & $\Delta_{3}$ \\
\hline$v_{\varepsilon}: 1 \leq \varepsilon \leq s+1$ & $t-s-1+\varepsilon$ & $t-s+\varepsilon-2$ & 0 \\
$v_{\varepsilon}: s+2 \leq \varepsilon \leq t+s+1$ & $t+s-\varepsilon+1$ & $t+s-\varepsilon+2$ & $a$ \\
$v_{\varepsilon}: t+s+2 \leq \varepsilon \leq n$ & $\varepsilon-t-s-1$ & $\varepsilon-t-s-2$ & $a$ \\
\hline
\end{tabular}

Case 2.2: when $n=2 t+1$ and $m=2 s$, where $a=1$ when $\varepsilon=t+s+1$ and $t+s+2$ and 0 , otherwise. Table 17 shows different representations of $v_{\varepsilon}$ with respect to the partition $\Delta$.

Since all the vertices of $C_{n}^{m}$ have different representations with respect to the partition $\Delta$, therefore,

$$
\operatorname{pd}\left(C_{n}^{m}\right) \leq 3
$$

Conversely. To prove that $\operatorname{pd}\left(C_{n}^{m}\right) \geq 3$, suppose, on the contrary, $\operatorname{pd}\left(C_{n}^{m}\right)=2$. It is not possible because $\operatorname{pd}(G)=2$ if and only $G$ is a path graph. Therefore,

$$
\operatorname{pd}\left(C_{n}^{m}\right) \geq 3
$$

Hence, from inequalities (5) and (6), we can conclude that 
TABLE 17: Distance codes for the vertices of $C_{n}^{m}$ w.r.t $\Delta$.

\begin{tabular}{lccc}
\hline$d(\cdot, \cdot)$ & $\Delta_{1}$ & $\Delta_{2}$ & $\Delta_{3}$ \\
\hline$v_{\varepsilon}: 1 \leq \varepsilon \leq s$ & $t-s+\varepsilon$ & $t-s+\varepsilon-1$ & 0 \\
$v_{\varepsilon}: \varepsilon=s+1$ & $t$ & $t$ & 0 \\
$v_{\varepsilon}: s+2 \leq \varepsilon \leq t+s+1$ & $t+s-\varepsilon+1$ & $t+s-\varepsilon+2$ & $a$ \\
$v_{\varepsilon}: t+s+2 \leq \varepsilon \leq n$ & $\varepsilon-t-s-1$ & $\varepsilon-t-s-2$ & $a$ \\
\hline
\end{tabular}

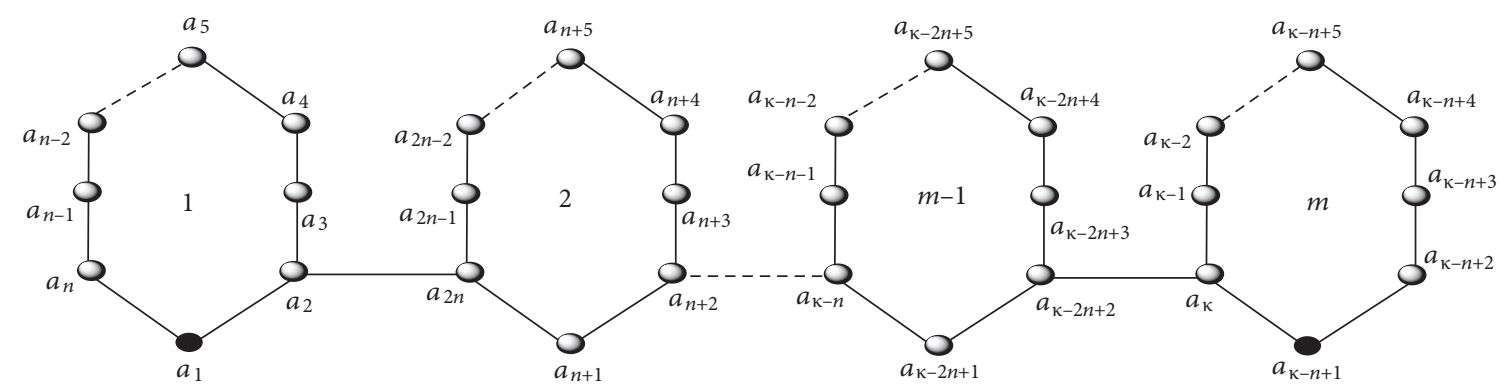

FIgURE 3: Chain of cycles' graph $\mathrm{CC}_{n}^{m}$.

TABle 18: Distance codes for $a_{k}$ w.r.t $\Delta_{1}$.

\begin{tabular}{lc}
\hline$d(\cdot, \cdot)$ & $\Delta_{1}$ \\
\hline$a_{k}: k=1,2,3, \ldots,\lceil(n+1 / 2)\rceil$ & $k-1$ \\
$a_{k}: k=\lceil(n+1 / 2)\rceil+1,\lceil(n+1 / 2)\rceil+2, \ldots, n$ & $n-k+1$ \\
$a_{k}: k \equiv 1,2,3, \ldots,\lfloor(n-1 / 2)\rfloor(\bmod n)$ and $k \geq n+1$ & $3\lfloor(k / n)\rfloor+k-1-n\lfloor(k / n)\rfloor$ \\
$a_{k}: k \equiv 0,\lfloor(n-1 / 2)\rfloor+1,\lfloor(n-1 / 2)\rfloor+2, \ldots, n-1(\bmod n)$ and & $n\lfloor(k / n)\rfloor+2-k$ \\
$n+1 \leq k \leq 2 n$ & \\
$a_{k}: k \equiv 0,\lfloor(n-1 / 2)\rfloor+1,\lfloor(n-1 / 2)\rfloor+2, \ldots, n-1(\bmod n)$ and & $n\lfloor(k+\lfloor(n-1 / 2)\rfloor+1 / n)\rfloor-k+2+3(\lfloor(k+\lfloor(n-1 / 2)\rfloor+1 / n)\rfloor-2)$ \\
$k>2 n$ &
\end{tabular}

TABLE 19: Distance codes for $a_{k}$ w.r.t $\Delta_{2}, m=2$.

\begin{tabular}{lr}
\hline$d(\cdot, \cdot)$ & $\Delta_{1}$ \\
\hline$a_{k}: k=1$ & 3 \\
$a_{k}: k=2,3, \ldots,\lfloor(n+2 / 2)\rfloor$ & $k$ \\
$a_{k}: k=\lfloor(n+2 / 2)\rfloor+1, \ldots, n$ & $n-k+4$ \\
\hline
\end{tabular}

TABLe 20: Distance codes for $a_{k}$ w.r.t $\Delta_{2}, m \geq 3$.

\begin{tabular}{lr}
\hline$d(\cdot, \cdot)$ & $\Delta_{2}$ \\
\hline$a_{k}: k=1$ & $3(\lfloor(n(m-1)+1 / n)\rfloor-1)+k$ \\
$a_{k}: k=2,3, \ldots,\lfloor(n+2 / 2)\rfloor$ & $3(\lfloor(k / n)\rfloor-1)+k$ \\
$a_{k}: k=\lfloor(n+2 / 2)\rfloor+1, \ldots, n$ and $k \geq n+1$ & $n-k+4+3(\lfloor(n(m-1)+1 / n)\rfloor-1)$ \\
\hline
\end{tabular}

$$
\operatorname{pd}\left(C_{n}^{m}\right)=3
$$

\section{Cycles' Chain Graph}

This section deals with the graph generated by taking and joining $m$ copies of cycles and $m-1$ copies of path of length 1 alternately. This graph is denoted by $\mathrm{CC}_{n}^{m}$ with vertex set $V(G)=\left\{a_{1}, a_{2}, \ldots, a_{m n}\right\}$ and edge set $E(G)=\left\{a_{i} a\right.$ $\left.{ }_{i+1}: 1 \leq i \leq n\right\} \cup\left\{a_{(m-1) n+}\right.$

$\left.i a_{(m-1) n+i+1}: m \geq 2\right\} \cup\left\{a_{(j-1) n+2} a_{(j+1) n}: 1 \leq j \leq m-1\right\}$, where $m$ represents the number of cycles and $n$ represents the number of vertices in a cycle, and for each cycle, $a_{m n+1}=a_{(m-1) n+1}$, i.e., for $m=1$ and $a_{n+1}=a_{1}$ and for $m=2 a_{2 n+1}=a_{n+1}$. The order and size of the chain graph is $\kappa=\left|V\left(\mathrm{CC}_{n}^{m}\right)\right|=n m$ and $\left|E\left(\mathrm{CC}_{n}^{m}\right)\right|=m(n+1)-1$, respectively. Figure 3 shows chain of cycles' graph $\mathrm{CC}_{n}^{m}$.

4.1. Partition Dimension of Chain of Cycles' Graph $C C_{n}^{m}$. In this section, we study the partition dimension of graph generated by chain of cycles $\mathrm{CC}_{n}^{m}$. The following theorem presents the partition dimension of the graph $\mathrm{CC}_{n}^{m}$. 
TABLE 21: Distance codes for $a_{k}$ w.r.t $\Delta_{2}, m \geq 2$.

\begin{tabular}{lc}
\hline$d(\cdot, \cdot)$ & $\Delta_{2}$ \\
\hline$a_{k}: k=n(m-1)+1, n(m-1)+2, \ldots, n(m-1)+\lfloor(n / 2)\rfloor$ & $k-n(m-1)-1$ \\
$a_{k}: k=n(m-1)+\lfloor(n / 2)\rfloor+1, n(m-1)+\lfloor(n / 2)\rfloor+2, \ldots, n m$ & $n m-k+1$ \\
$a_{k}: k \equiv 2,3, \ldots,\lfloor(n+2 / 2)\rfloor(\bmod n)$ and $n+1 \leq k \leq n(m-1)$ & $3(m-\lfloor(k-1 / n)\rfloor-2)+k-n\lfloor(k / n)\rfloor$ \\
$a_{k}: k \equiv 0,\lfloor(n+2 / 2)\rfloor+1, \ldots, n-1(\bmod n)$ and $n+1 \leq k \leq n(m-1)$ & $3(m-\lfloor(k-1 / n)\rfloor-2)+4-k+n\lfloor(k+\lfloor(n+2 / 2)\rfloor / n)\rfloor$ \\
$a_{k}: k \equiv 1(\bmod n)$ and $n+1 \leq k \leq n(m-1)$ & $3(m-\lfloor(k / n)\rfloor-1)$ \\
\hline
\end{tabular}

Theorem 5. If $n \geq 5$ and $m \geq 2$, then $p d\left(C C_{n}^{m}\right)=3$.

Proof. First, we prove $\mathrm{pd}\left(\mathrm{CC}_{n}^{m}\right) \leq 3$ by constructing a resolving partition set $\Delta=\left\{\Delta_{1}, \Delta_{2}, \Delta_{3}\right\} \Delta_{1}=\left\{a_{1}\right\}$, $\Delta_{2}=\left\{a_{\kappa-n+1}\right\}$, and $\Delta_{3}=\left\{a_{k} \in V(G) \mid a_{k} \notin \Delta_{1}, \Delta_{2}\right\}$ from the vertex set of $\mathrm{CC}_{n}^{m}$. We assume the following cases on the vertex set of $G=\mathrm{CC}_{n}^{m}$ and on the copies of cycle graph, i.e., $m$.

Table 18 shows different representations of $a_{k}$ with respect to the partition $\Delta_{1}$.

Tables 19-21 show different representations of $a_{k}$ with respect to the partition $\Delta_{2}$.

Third vector representations are

$$
r\left(a_{k} \mid \Delta_{3}\right)= \begin{cases}1, & k=1, \kappa-n+1, \\ 0, & \text { otherwise. }\end{cases}
$$

Hence, it follows from the above discussion that $\operatorname{pd}\left(\mathrm{CC}_{n}^{m}\right) \leq 3$ because all the vertices of $\mathrm{CC}_{n}^{m}$ have unique representations with respect to resolving partition set $\Delta$.

The reverse inequality pd $\left(\mathrm{CC}_{n}^{m}\right) \geq 3$ can be easily followed from the fact that partition dimension of the graph $G$ is 2 if and only if $G$ is a path graph. Thus, we conclude that, for $n \geq 5$ and $m \geq 2$,

$$
\operatorname{pd}\left(\mathrm{CC}_{n}^{m}\right)=3
$$

\section{Conclusion and Discussion}

In this paper, we computed partition dimension of cycle-related graph such as paddle graph and cycle graph with chord and chain of cycles' graph. It has been shown that partition dimension of the aforementioned graph is 3 . It was proved that the partition dimension of the cycle graph [1] is 3 . We conclude that, by making small or significant changes in the cycle graph, do not affect its partition dimension.

\section{Data Availability}

No data were used to support the study.

\section{Conflicts of Interest}

The authors declare no conflicts of interest.

\section{Authors' Contributions}

All the authors contributed equally for the preparation of this article.

\section{Acknowledgments}

This paper was supported by Anhui Natural Science Research Project (2020) under Grant no. KJ2020A0696.

\section{References}

[1] G. Chartrand, E. Salehi, and P. Zhang, "The partition dimension of a graph," Aequationes Mathematicae, vol. 59, no. 1 , pp. $45-54,2000$.

[2] F. Harary and R. A. Melter, "On the metric dimension of a graph," Ars Combinatoria, vol. 2, pp. 191-195, 1976.

[3] P. J. Slater, "Leaves of trees," Congress Numerantium, vol. 14, pp. 549-559, 1975.

[4] M. R. Garey and D. S. Johnson, Computers and Intractability: A Guide to the Theory of NP-Completeness, Freeman, New York, NY, USA, 1979.

[5] N. Mehreen, R. Farooq, R. Farooq, and S. Akhter, "On partition dimension of fullerene graphs," AIMS Mathematics, vol. 3, no. 3, pp. 343-352, 2018.

[6] M. C. Monica and S. Santhakumar, "Partition dimension of certain honeycomb derived networks," International Journal of Pure and Applied Mathematics, vol. 108, no. 4, pp. 809-818, 2016.

[7] B. Rajan, A. William, I. Rajasingh, C. Grigorious, and S. Stephen, "On certain networks with partition dimension three," in Proceedings of International Conference on Mathematics Engineering and Business Management, Chengdu, China, March 2012.

[8] J. A. Rodríguez-Velázquez, I. Lemańska, and M. Lemanska, "On the partition dimension of trees," Discrete Applied Mathematics, vol. 166, pp. 204-209, 2014.

[9] J. A. Rodrìguez-Velàzquez, I. G. Yero, and H. Fernau, "On the partition dimension of unicyclic graphs," Bulletin Mathematics Society of Science and Mathematics, vol. 57, pp. 381-391, 2014.

[10] R. Juan, I. G. Yero, and M. Lemanska, "On the partition dimension of trees," Discrete Applied Mathematics, vol. 166, pp. 204-209, 2014

[11] H. A. Amrullah and E. T. Baskoro, "The partition dimension for a subdivision of homogeneous caterpillars," AKCE International Journal of Graphs and Combinatorics, vol. 130, pp. 157-168, 1998

[12] D. Amrullah and E. T. Baskoro, "The partition dimension for a homogeneous firecrackers," Far East Journal of Applied Mathematics, vol. 90, no. 1, pp. 77-98, 2015.

[13] H. M. A. Siddiqui and M. Imran, "Computation of metric dimension and partition dimension of Nanotubes," Journal of Computational and Theoretical Nanoscience, vol. 12, no. 2, pp. 199-203, 2015.

[14] J. Cáceres, C. Hernando, M. Mora et al., "On the metric dimension of Cartesian products of graphs," SIAM Journal on Discrete Mathematics, vol. 21, no. 2, pp. 423-441, 2007. 
[15] V. Chvatal, Mastermind, Combinatorica, vol. 3, no. 3-4, pp. 325-329, 1983.

[16] Z. Beerliova, F. Eberhard, T. Erlebach et al., "Network discovery and verification," IEEE Journal on Selected Areas in Communications, vol. 24, no. 12, pp. 2168-2181, 2006.

[17] M. A. Johnson, "Structure-activity maps for visualizing the graph variables arising in drug design," Journal of Biopharmacy and Statistics, vol. 3, pp. 203-236, 1993.

[18] M. A. Johnson, "Browsable structure-activity datasets," Advances in Molecular Similarity, pp. 153-170, JAI Press Connecticut, Stamford, CT, USA, 1998.

[19] R. A. Melter and I. Tomescu, "Metric bases in digital geometry," Computer Vision, Graphics, and Image Processing, vol. 25, no. 1, pp. 113-121, 1984.

[20] G. Chartrand, L. Eroh, M. A. Johnson, and O. R. Oellermann, "Resolvability in graphs and the metric dimension of a graph," Discrete Applied Mathematics, vol. 105, no. 1-3, pp. 99-113, 2000.

[21] A. Ahmad, M. Bača, and S. Sultan, "Computing the metric dimension of kayak paddle graph and cycles with chord," Proyecciones Journal of Mathematics, vol. 39, no. 2, pp. 287-300, 2020.

[22] I. Javaid and S. Shokat, "On the partition dimension of some wheel related graphs," Journal of Prime Research in Mathematics, vol. 4, pp. 154-164, 2008.

[23] Y.-M. Chu, M. F. Nadeem, M. Azeem, and M. K. Siddiqui, "On sharp bounds on partition dimension of convex polytopes," IEEE Access, vol. 8, Article ID 224781, 2020.

[24] S. Pirzada, M. Aijaz, and S. P. Redmond, "Upper dimension and basis of zero divisor graphs of commutative rings," AKCE International Journal of Graphs and Combinatorics, vol. 17, pp. 168-173, 2019.

[25] S. Pirzada, M. Aijaz, and S. P. Redmond, "On upper dimension of graphs and their bases sets," Discrete Mathematics Letter, vol. 3, pp. 37-43, 2020.

[26] S. Pirzada and M. Aijaz, "On graphs with same metric and upper dimension," Discrete Mathematics, Algorithms and Applications, vol. 13, no. 02, Article ID 2150015, 2021. 